\section{Revival of an old technique}

from A. I. Ferguson

THE optoacoustic or photoacoustic effect, an intriguing example of which is described ${ }^{1}$ on p.321 of this issue of Nature, is proving an important and sensitive technique for studying the interaction of light with matter. The effect was discovered over one hundred years ago by Alexander Graham Bell ${ }^{2}$ but, after a period of rather intense study when an attempt was made to develop a 'photophone' (see inset), the effect was dismissed as a curiosity. In the past decade there has been a revival of interest in optoacoustic phenomena, and now, with the availability of lasers and the development of sensitive microphones, the optoacoustic effect can be used as a valuable probe in such diverse scientific fields as physics, chemistry, biology and medicine ${ }^{3,4}$. There is also a growing interest in applying the techniques in the food and drug industries and in agricultural science.

In Bell's original observation, sunlight was focused on to a selenium photocell. Although Bell was primarily interested in detecting the electrical changes in the selenium cell as the sunlight was chopped, he found that sound at this chopping frequency could be heard by a nearby observer. Modern optoacoustic experiments bear a striking resemblance to this. The modern light source is more likely to be an intense arc lamp or laser and the acoustic signals are detected by having an exchange gas which couples the sample and the microphone. Heating and cooling of the sample heats and cools the gas and the resulting changes in pressure are detected in the microphone. In his book Photoacoustics and Photoacoustic Spectroscopy ${ }^{4}$, Rosencwaig reviews many applications of this method in samples ranging from single crystals through powders of inorganic insulators to biological tissue. A useful feature of the gas microphone technique is that by varying the chopping frequency of the light it is possible to obtain depth profile information about the sample.

In the experiments described in this issue of Nature ${ }^{1}$, O'Connor and Diebold have detected absorption in $\mathrm{Cl}_{2} / \mathrm{H}_{2} / \mathrm{N}_{2}$ mixtures using a gas microphone. By shining an argon ion laser at $476 \mathrm{~nm}$ into the gas mixture they find an enhancement - of about $10^{3}$ - of the optoacoustic signal over that obtained when $\mathrm{H}_{2}$ is absent. The enhancement has been attributed to the photodissociation of $\mathrm{Cl}_{2}$ followed by a chain reaction involving $\mathrm{H}_{2}$ and $\mathrm{Cl}$. Thus a small amount of laser energy releases a large amount of chemical energy. Indeed, the authors report that explosions take place when the laser power exceeds about $10 \mathrm{~mW}$ - this must be the ultimate in opto-

\section{acoustic detection!}

By monitoring the optoacoustic signal as a function of time it is possible to observe the concentration of the reactants as the reaction takes place. A tantalizing possibility is that the speed of the chain reaction could be monitored by observing the optoacoustic signal as a function of chopping frequency. Although the authors suggest such investigations they have not yet carried them out.

Optoacoustic detection is by no means limited to the use of conventional microphones. Indeed, some of the smallest detected absorptions have been measured using other methods - for example, detection of direct strain measurements and detection of refractive index gradients.

In the direct strain method, a piezoceramic is bonded to the sample. Strains induced in the sample by the absorbed radiation can be directly measured as a voltage across the piezoceramic. Absorptions of as small as 1 part in $10^{7}$ have been detected using this technique when the sample is illuminated with a pulsed dye laser ${ }^{5}$.

Träger et $a l .{ }^{6}$ have recently shown that the method can be used to study surface interactions. Using a silver-coated piezoceramic transducer they studied the absorption spectrum of $\mathrm{SF}_{6}$ laid down on the silver surface. They have found that they can detect surface adsorption of $\mathrm{SF}_{6}$ down to 0.1 of a monolayer. These studies could have important implications for the semiconductor fabrication industry.

Techniques based on the detection of a refractive index gradient have the advantage that they involve no direct contact and so may be used on very hostile samples, for example, plasmas or flames. In this case the local heating and cooling causes local changes in the refractive index of the sample. A second light source, usually a low-power laser, then probes the excitation region. The gradient of the refractive index causes changes in the direction of the probe beam which can be easily detected. A recent publication by Zapka et al. ${ }^{7}$ has demonstrated the use of this method in spatially resolving and measuring the temperature of a flame by measuring the arrival time of an acoustic pulse. The technique could be applied to observing other forms of combustion.

The range of potential applications of optoacoustic detection is enormous. Many of the examples of optoacoustic phenomena described in the literature have simply been demonstrations. As the method matures and is more widely appreciated it will inevitably become an indispensable tool for the laboratory scientist in disciplines ranging from the esoteric to the highly practical. Many of these new applications will be explored at the 'Third International Toptical Meeting on Photoacoustic Spectroscopy' which is to be held in Aussois, France in May.

A. I. Ferguson is in the Department of Physics, The University, Southampton SO9 SNH.

1. O'Connor, M.T. \& Dicbold, G.J. Nature 301, 321 (1983) 2. Bell, A.G. Proc. Am. Ass. Advmt Sci. 29, 115 (1880).

3. Pao, Y-H. Optoocoustic Spectroscopy and Detection (Academic, New York, 1977).

4. Rosencwaig, A. Photoacoustics and Photoacoustic Spectroscopy (Wiley, New York, 1980)

5. Patel, C.K.N. \& Tam. A.C. Rev.Mod.Phys. 53, 517 (1981).

6. Trăger, F., Confal, H. \& Chuang, T.J. Phys.Rev.Lett. 49. 1720 (1982)

7. Zapka, W., Pokrowsky, P. \& Tam, A.C. Opt. Lett. 7, 477 (1982).

\title{
BELL'S PHOTOPHONE
}

OuR readers are already aware that the object of the photophone is the transmission of sounds both musical and vocal to a distance by the agency of a beam of light of varying intensity .... not the least of the remarkable points in this research is the discovery that audible vibrations are set up in thin disks of almost every kind of material by merely throwing upon them an intermittent light. Hence in theory, if not in practice, the receiver may be reduced to the divine simplicity of a mere disk of vulcanite or of zinc, on one side of which the vibrating beam of light falls, and at the other side of which the hearer listens.

We may remind our readers here that this apparent direct conversion of light into sound takes place, as Prof. Bell found, in disks of all kinds of substances - hard rubber, zinc, antimony, selenium, ivory, parchment, wood, and that he has lately found that disks of carbon and of thin glass, which he formerly thought exceptions to this property, do also behave in the same way.

We may perhaps remark without impropriety that it is extremely improbable that the apparent conversion of light into sound is by any means a direct process. It is well known that luminiferous rays, when absorbed at the surface of a medium, warm that surface slightly, and must therefore produce physical and molecular actions in its structure. If it can be shown that this warming effect and an intermediate cooling by conduction can go on with such excessive rapidity that beams of light falling on the surface at intervals less than the hundredth of a second apart produce a discontinuous molecular action of alternate expansion and contraction, then the mysterious property of matter revealed by these experiments is accounted for.

As reported in Nature, 4 November 1880. 